\title{
Skin tests and challenge-based drug allergy diagnosis: a retrospective study of patients with confirmed drug allergy
}

\author{
${ }^{1}$ CINTESIS, Center for Health Technology and Services Research, Faculty of Medicine, University of Porto, Portugal \\ ${ }^{2}$ Allergy Unit, CUF-Porto Instituto and Hospital, Porto, Portugal \\ ${ }^{3}$ MEDIDA, Lda, Porto, Portugal \\ ${ }^{4}$ Basic and Clinical Immunology, Pathology Department, Faculty of Medicine, University of Porto, Porto, Portugal
}

\section{KEY WORDS}

drug allergy; drug challenge test; skin tests; predictors; decision tree

\section{Corresponding author}

Ana Margarida Pereira

Center for Research in Health Technologies

and Information Systems (CINTESIS)

Faculty of Medicine, University of Porto

Rua Plácido da Costa s/n

4200-450 Porto, Portugal

Phone: +351960 208480

E-mail: ambrpereira@gmail.com

\section{Doi}

10.23822/EurAnnACI.1764-1489.107

\begin{abstract}
Summary
Objectives. To describe clinical manifestations and performed diagnostic workup, focusing on drug challenge tests (DCT), in patients with drug allergy. Methods. Retrospective study including all patients with skin tests (STS) or DCT-based drug allergy diagnosis between 01/2014 - 06/2018 in a Portuguese allergy unit. Data were collected from electronic and paper-based clinical records. Results. We had 75 drug allergy diagnoses. Most index reactions were mild and $\geq 1$ hour after drug intake. Fifty-nine (78\%) diagnoses were based on DCTs, all based on multistep protocols with $\geq 3$ predicted steps. Only 10\% of the DCT were positive during up-dosing; timing and severity of the index reaction predicted DCT interruption during up-dosing. Conclusions. Most drug allergy diagnoses were based on multistep DCT. The identified predictors of DCT interruption during up-dosing can support the development of more personalized DCTs protocols.
\end{abstract}

\section{Introduction}

The term "drug allergy" is widely used in a popular sense to encompass both some type A reactions, which are predictable side effects due to the drug's pharmacological action, and type $\mathrm{B}$ reactions, which represent true hypersensitivity due to idiosyncratic and individual predisposition (1). However, the definite classification of a drug hypersensitivity reaction (DHR) is important for determining appropriate diagnostic procedures; immunological drug reactions can be divided into two broad types, as recommended by the World Allergy Organization (WAO) (1). These categories are based on the timing of the symptoms' onset: immediate DHRs occur within the first hours of the first administered dose and are usually IgE-mediated, while nonimmediate DHRs occur anytime thereafter; most of these reactions are cell-mediated hypersensitivities and involve several unknown mechanisms, which act simultaneously or even sequentially. However, the precise cut-off to differentiate immediate from nonimmediate reactions is controversial (2-5).

DHRs are often self-reported as "drug allergy" and not confirmed by appropriate assessment. This is a frequent problem in daily clinical practice and has a considerable impact on prescription choices and patient health. In fact, many more patients suspect they have a DHR than can be confirmed, indicating the importance of an accurate diagnosis of DHRs, which will facilitate appropriate treatment options and preventive measures (6). If the reaction is A-type, it is likely that the primary care physician will be able to manage it within the practice, however if it is a B-type reaction, it will require a structured diagnostic process by an allergy specialist $(6,7)$. The diagnostic approach to DHRs may include a detailed clinical history, followed by skin tests (ST), in vitro tests, and drug challenge tests (DCT). 
Since clinical history can be unreliable, the sensitivity of in vitro tests may be suboptimal and ST are not feasible nor validated for all drugs, a definitive diagnosis of drug hypersensitivity frequently relies on DCT (8). This test (double blinded, placebo controlled) is widely considered the gold standard for establishing or ruling out drug hypersensitivity. Moreover, it is also of major importance for assessing tolerance to potentially cross-reactive drugs and for providing alternative drugs (8). DCTs involve the controlled administration of a drug under medical surveillance. Therefore, it is a time-consuming and costly exam. A base-case penicillin allergy evaluation including skin testing followed by DCT is estimated to cost $\$ 220$ (9). Evaluation of all Americans who report penicillin allergy would cost over $\$ 7$ billion using this protocol (9-11).

There are a few published protocols for DCTs, but there is lack of information on the best protocol to use with each patient. In clinical practice, the diagnostic approach taken is highly inconsistent despite efforts from the scientific community to create clear-cut algorithms. In most European countries the diagnostic assessment takes place in specialized centers and is adapted depending on the drug involved and the type of allergic reaction suspected (e.g. immediate or non-immediate) (6). Different protocols have been described for immediate and nonimmediate DHRs, with some studies reporting increased diagnostic accuracy when prolonged DCTs are used in individuals with nonimmediate DHRs reactions (12-16). However, even in these nonimmediate DHRs tested with prolonged DCT protocols and having mean reaction delays $>48$ hours $(17,18)$, most studies report long first day, office-based, supervised oral DCTs, with multiple increasing doses that could possibly be shortened; this would result in decreased utilization of time and resources. Studies describing diagnostic procedures that confirmed drug allergy and reporting the predictors of DCT positivity during up-dosing are lacking; however, this could give valuable data to inform if shorter protocols for DCT could be safely used, at least in some patients.

With the present study, we aimed to: 1) describe drug allergy manifestations in patients with ST or DCT-based drug allergy diagnosis; 2) describe the diagnostic procedures that were performed to objectively diagnose drug allergy, focusing DCTs; and 3) estimate the proportion of DCTs interrupted before reaching the target cumulative dose, to describe the reacting dose/step and to make an exploratory analysis of the predictors of DCT interruption during up-dosing.

\section{Material and methods}

\section{Study design and data collection}

This was an observational, retrospective study held in a private allergy unit from Northern Portugal. All patients that had drug allergy confirmed by ST or DCTs between January 2014 and
June 2018 were eligible for inclusion. Patients with allergic contact dermatitis to drugs were excluded.

Data were collected from the electronic medical records, the final patient report and the specific DCT paper-forms that are used in the usual clinical practice for data registry; these paper forms include the prespecified protocol, doses administered, the symptoms and objective clinical manifestations arising during DCTs, and DCT outcome. Data was collected considering the variables suggested in the ENDA questionnaire (19).

All patients gave written informed consent to perform the diagnostic procedures. The collected data was anonymized before analysis.

\section{Diagnostic procedures}

The drug allergy workup was performed according to the physician's judgment, based on the EAACI guidelines on drug allergy diagnosis (4) and adapted according to the patient's history. The approach to a patient with suspected drug allergy involves a thorough characterization of the index reaction by the physician and, eventually, performing ST (skin prick tests, intradermal tests, patch tests), in vitro tests (e.g. specific IgE), and DCTs.

ST were done and interpreted according to the recommendations from Brockow et al. (20). ST were predominantly performed with drugs that have published validated concentrations (e.g. betalactams). However, in a few patients, especially in those who presented severe immediate index reactions, ST were performed with drugs without fully validated ST concentrations; in these cases, a literature review was performed prior to ST and the tested concentrations were selected according to the best available evidence. In patients with compatible index reaction performing ST with previously published non-irritant drug concentrations (even if not fully validated), especially if the suspected drug was not amenable to DCT, positive ST were considered sufficient to diagnose drug allergy. ST were not routinely performed when the suspected mechanism underlying the index reaction was not suggestive of being amenable to study with ST (e.g. most reactions with NSAIDs) (4).

DCTs were performed using multistep protocols, with administration of progressively higher doses of the suspected or alternative drug. DCT were continued until the therapeutic dose was reached, an objective adverse reaction arisen, or the patient revoked consent for the procedure. In our unit, all DCT are performed under medical supervision in a day ward setting. The usual DCT protocol depends on the drug and can be adapted according to the patient history or symptoms during the challenge; when available, previously published protocols were preferred. All DCT included an extended watching period with length adapted to the severity and time of onset of the index reaction. Patients with late reactions described as maculopapular exanthems had extended DCTs (at least three days of outpatient 
A.M. Pereira, M. Couto, M. Pereira, L. Araújo

drug intake), according to the findings of some recent studies, both in pediatric $(12,13,18,21)$ and adult $(12,14-16)$ populations. Most patients with late reactions described as maculopapular exanthems (e.g. to betalactams), performed DCT without previous ST (12).

DCTs were considered positive based on the presence of objective signs and reproducibility between DCT and index reaction. When the patient presented no objective signs or nonreproducible minor symptoms, the DCT was considered inconclusive and was not included in this analysis.

Patients with severe cutaneous manifestations $(4,22)$, including Stevens-Johnson syndrome (SJS), toxic epidermal necrolysis (TEN), acute generalised exanthematous pustulosis (AGEP), vasculitis or drug reaction with eosinophilia and systemic symptoms (DRESS) were not considered for diagnostic DCT.

\section{Variables and classifications}

The up-dosing phase of the DCT was considered completed if the predefined target cumulative dose (at least one therapeutic dose of the specific drug) was reached. When this dose was not attained, the up-dosing phase was considered as interrupted.

Cutaneous drug hypersensitivity reactions were classified according to the EAACI recommendations (22).

The severity of systemic reactions was classified according to the classification proposed by Cox et al. (23), including 5 different grades. This classification system intends to be a common way to describe the severity of systemic allergic reactions, applicable not only to immunotherapy (as the WAO classification system (24)), but also to drug, food or venom allergy. However, it seems primarily designed to classify severity of immediate reactions and does not clearly state that can be applied to delayed DHR. In this study, this classification system was used irrespectively of the time of reaction onset.

The cut-off to define immediate and nonimmediate drug allergy is still controversial: Levine et al. (5) defined immediate reactions as those beginning $<2$ hours after drug intake, accelerated between 2 and $48 \mathrm{~h}$, and delayed after 48 hours; Romano et al. (2) considered immediate beginning $\leq 1$ hour and non-immediate $>1$ hour; other authors $(3,4,25)$ reported that immediate reactions can begin until 6 hours of drug intake and nonimmediate anytime thereafter. To perform exploratory analysis including all these different possible cut-offs, we classified the timing of index and DCT reactions into 5 classes $(\leq 1$ hour, 1 to 2 hours, 2 to 6 hours, 6 to 48 hours, and $>48$ hours).

\section{Statistical analysis}

Data was analysed by patient and by drug allergy diagnosed. In patients with more than one reaction to the same drug, the most recent was included in the descriptions.
Categorical data was described with absolute and relative frequencies and continuous variables (age) with mean and standard deviation (SD). Pearson chi-square was used for comparison of proportions when all categories had $\mathrm{n}>5$; in very small groups (at least one category with $\mathrm{n} \leq 5$ ) a 2-sided Fisher's exact test was used. Variables organized as trend were analysed with linear-by-linear association chi-square. Comparisons of continuous variables, due to the small sample size and variable distribution, were performed using non-parametric tests, including Kruskal-Wallis (for k independent samples) and Mann Whitney U (for 2 independent samples).

Agreement between categorical variables was assessed with Cohen's kappa $(\mathrm{K})$. The $\mathrm{K}$ value was interpreted as follows (26): $<0.20$ poor; 0.20 to 0.40 fair; 0.41 to 0.60 moderate; 0.61 to 0.80 good; 0.81 to 1.00 very good.

We used CART (classification and regression tree) analysis to identify the most important predictors of DCT outcome during up-dosing. CART is a nonparametric supervised classification method that is intended as an exploratory tool to discover homogeneous subgroups within the data; it is appealing because of the apparent closeness to the human reasoning processes, presenting data in easy to interpret tree models (27). We performed CART analysis using Gini impurity index to grow the trees and the cost-complexity pruning algorithm to generate a simpler tree. The chosen result is within one standard error of the tree with best error estimate, favouring trees with minimum costs. The predictors included in CART were selected based on an unadjusted analysis that identified the variables significantly associated with DCT outcome during up-dosing. The variable importance was given by Gini index, where the highest value is $100 \%$. Variables that did not contribute significantly to the model were automatically removed. CART analysis was performed with Salford Predictive Model$\mathrm{er}^{\circledR}$ version 8.2 (Salford Systems, San Diego, USA). All other data analyses were performed with IBM SPSS ${ }^{\circledR}$ version 25 (IBM Corporation, Armonk, USA). P-values $<0.05$ were defined as statistically significant.

\section{Results}

We performed 589 drug allergy workups, including 528 DCT and 179 sessions of ST, in 496 patients. During the study period, we had $72(15 \%)$ patients with drug allergy diagnosis confirmed with ST or DCT. Seventy one percent $(n=51)$ were female with a mean (SD) age of 34 (21) years; 21 (29\%) were < 18 years.

Two patients had confirmed allergy to more than one drug (maximum 3), totalling 75 drug allergies diagnosed.

\section{Characteristics of the index reactions}

The characteristics of the index reactions are described in table I. About half of the reactions were caused by betalactams ( $45 \%$, 
Table I - Characteristics of the index reactions, including stratification by the diagnostic procedure that confirmed drug allergy. When more than one reaction was reported for the same drug, the most recent was included. ST were performed in 31 (41\%) of the included reactions.

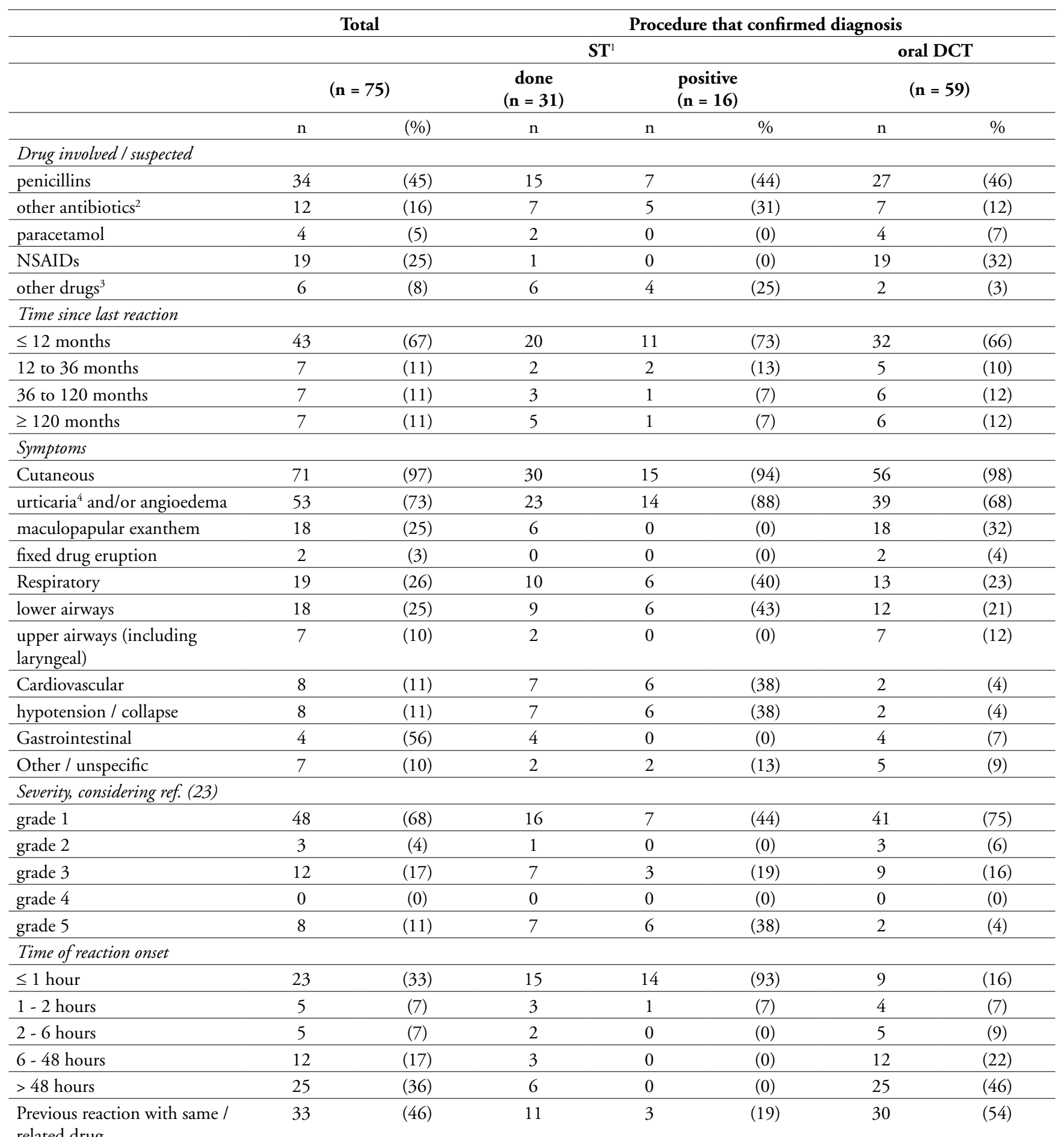
related drug

${ }^{1}$ Includes skin prick tests and intradermal tests; ${ }^{2}$ Including cephalosporins, ciprofloxacin, cotrimoxazole, levofloxacin, minocycline, nitrofurantoin, vancomycin; ${ }^{3}$ Including albendazole, atropine, betamethasone, cisatracurium, influenza vaccine, patent blue; ${ }^{4}$ Includes urticaria and/or erythema-warmth and/or pruritus, other than localized at the injection site (23). NSAID, nonsteroidal anti-inflammatory drug. 
A.M. Pereira, M. Couto, M. Pereira, L. Araújo

$\mathrm{n}=34$ penicillins, and $5 \%, \mathrm{n}=4$ cephalosporins). Two thirds of the patients had the last reaction to the culprit drug in the past 12 months and about half reported at least another previous reaction with the same or a related drug. Almost all patients reported cutaneous symptoms, one quarter respiratory involvement, and one tenth had hypotension and/or collapse; sixty-one percent $(n=46)$ of the reactions presented with exclusive cutaneous symptoms. Most reactions presented grade 1 severity; eight $(11 \%)$ were grade 5 . One third of the index reactions occurred in the first hour after drug intake. Two patients had no information regarding symptoms and timing of the index reaction: one child with asthma that had confirmed anaphylaxis to paracetamol and tested ibuprofen to exclude possible cross-reactivity; another patient did not remember any characteristic of the previous reaction.

\section{Description of the diagnostic procedures performed}

Two (3\%) drug allergies were confirmed by skin prick tests and 14 (19\%) by intradermal tests. Fifty-nine (78\%) diagnoses were made by oral DCT. None of these patients performed patch tests. Characteristics of the index reactions stratified by the diagnostic procedure that confirmed drug allergy are presented in table I. More than half of the ST were performed in patients with very immediate ( $<1$ hour) index reactions and almost all were positive. No patient with a reaction beginning more than 2 hours after drug intake had positive ST. Otherwise, $75 \%$ of the patients with grade 5 reactions had diagnosis confirmed by positive $S T$.

Fifty-four (92\%) DCTs were performed with diagnostic intent. The other five DCTs intended to find a suitable alternative within the class of nonsteroidal anti-inflammatory drugs (NSAID). Two of these patients presented chronic urticaria with multiple NSAID intolerance, including to selective COX2 inhibitors (meloxicam and etoricoxib); as both of them had indication to take NSAID due to comorbid diseases, we decided to perform the DCT under preventive treatment with anti-histamine \pm montelukast to increase the odds of finding a suitable alternative strategy that could give an answer to their need of NSAID intake. Nevertheless, even with this preventive strategy, the DCT were positive.

All DCTs were performed using multistep protocols, with at least three predicted steps (maximum 7 steps). The number of predicted steps was not significantly and independently associated with symptoms, severity or time of onset of the index reaction, but was significantly associated with the drug tested ( $\mathrm{p}=0.033$ ). DCTs with penicillins had the highest proportion of procedures with at least 6 predicted steps (65\% vs. $29 \%$ with other antibiotics, vs. $26 \%$ with NSAIDs vs. $0 \%$ with paracetamol and other drugs; $\mathrm{p}=0.013$ ).

\section{Description of DCTs results}

Six (10\%) DCTs were considered positive and interrupted during the up-dosing phase. In these DCTs, the reaction occurred at 4 to $45 \%$ of the predicted cumulative dose; two patients had positive DCT with less than $10 \%$ of the predicted dose. The proportion of missed steps ranged from 17 to $67 \%$ of the predicted. Ninety percent $(\mathrm{n}=53)$ of the DCTs had the up-dosing phase completed. Thirteen (22\%) were positive during the watching period and $40(68 \%)$ after day ward discharge (at least 5 hours after the last supervised dose intake). Thirty-four patients had extended DCT, maintaining drug intake in the days after discharge; the median time to reaction was 5.5 days (maximum 11 days). About two thirds $(\mathrm{n}=37)$ of the DCT reactions occurred within the same time period reported for the index. However, $15(27 \%)$ were faster during DCT. Five $(9 \%)$ reactions with index onset between 2 and 48 hours occurred within the first hour of the last drug intake during DCT. No reaction with index onset $>48$ hours had symptoms within the first 2 hours.

A description of the symptoms presented during the DCT is presented in table II. Almost all patients presented cutaneous symptoms, with a complete agreement with the index reaction. However, the specific type of cutaneous reaction had low agreement (except for fixed drug eruption). Cardiovascular symptoms were present in only one patient.

Seventy-two percent $(n=41)$ of the DCT reactions were grade 1 ; only one patient had a grade 5 reaction. Eighty percent $(\mathrm{n}=$ 44) of the DCT reactions were of similar severity grade as index reactions. Six (11\%) had higher severity during DCT; the largest variation (from a grade 1 index reaction to a grade 4 DCT reaction) occurred in one patient that reported a mild cutaneous reaction to penicillin more than 10 years before the DCT.

\section{Predictors of DCT outcome during up-dosing - exploratory analyses}

The characteristics of patients that interrupted DCT are presented in table III.

DCTs that were interrupted during up-dosing were performed with NSAIDs, paracetamol and levofloxacin; no penicillin DCT was positive/interrupted during up-dosing. All patients that interrupted DCT before reaching the cumulative dose reported lower airways symptoms $(\mathrm{p}<0.001)$ and very immediate index reactions $(\mathrm{p}<0.001)$ with at least grade 3 severity.

No significant associations were found between DCT outcome during up-dosing and gender $(\mathrm{p}=0.658)$, age group ( $\mathrm{p}$ $=0.653)$, time since last reaction $(\mathrm{p}=0.682)$, another or index reaction with cutaneous $(0.754)$, cardiovascular $(0.169)$ or gastrointestinal (0.315) symptoms.

All variables identified as being significantly associated with DCT outcome (table III) were included in the CART analysis 
Table II - Symptoms present at the DCT and comparison with the reported index reactions. Two patients had missing information regarding the index reaction.

\begin{tabular}{|c|c|c|c|c|c|c|c|c|c|c|c|}
\hline & & & \multicolumn{9}{|c|}{ Comparison with index reaction } \\
\hline & \multirow{2}{*}{\multicolumn{2}{|c|}{$\begin{array}{l}\text { Total } \\
(\mathrm{n}=59)\end{array}$}} & \multicolumn{4}{|c|}{ concordant } & \multicolumn{4}{|c|}{ discordant } & \multirow{3}{*}{ kappa } \\
\hline & & & \multicolumn{2}{|c|}{ present } & \multicolumn{2}{|c|}{ absent } & \multicolumn{2}{|c|}{ present in index } & \multicolumn{2}{|c|}{ present in dct } & \\
\hline & $\mathrm{n}$ & $(\%)$ & $\mathrm{n}$ & $\%$ & $\mathrm{n}$ & $\%$ & $\mathrm{n}$ & $\%$ & $\mathrm{n}$ & $\%$ & \\
\hline Cutaneous & 58 & (98) & 56 & (98) & 1 & $(2)$ & 0 & (0) & 0 & $(0)$ & 1.000 \\
\hline urticaria ${ }^{1}$ and/or angioedema & 36 & $(61)$ & 20 & $(35)$ & 15 & (26) & 8 & (14) & 14 & $(25)$ & 0.231 \\
\hline maculopapular exanthem & 26 & $(44)$ & 16 & $(28)$ & 29 & $(51)$ & 2 & $(4)$ & 10 & $(18)$ & 0.565 \\
\hline fixed drug eruption & 2 & (3) & 2 & $(4)$ & 55 & (97) & 0 & (0) & 0 & $(0)$ & 1.000 \\
\hline Respiratory & 16 & (27) & 11 & (19) & 41 & $(72)$ & 2 & $(4)$ & 3 & $(5)$ & 0.757 \\
\hline lower airways & 6 & (10) & 5 & (9) & 45 & (79) & 7 & (12) & 0 & $(0)$ & 0.530 \\
\hline $\begin{array}{l}\text { upper airways (including } \\
\text { laryngeal) }\end{array}$ & 12 & (20) & 5 & (9) & 45 & (79) & 2 & $(4)$ & 5 & (9) & 0.519 \\
\hline Cardiovascular & 1 & (2) & 0 & (0) & 54 & (95) & 2 & $(4)$ & 1 & (2) & 0.024 \\
\hline Gastrointestinal & 1 & (1) & 1 & (2) & 53 & (93) & 3 & (5) & 0 & $(0)$ & 0.383 \\
\hline
\end{tabular}

${ }^{1}$ Includes urticaria and/or erythema-warmth and/or pruritus, other than localized at the injection site (23).

Table III - Patients' characteristics according to the DCT outcome during the up-dosing phase (interrupted vs. completed). Two patients had no information regarding the symptoms of index reaction.

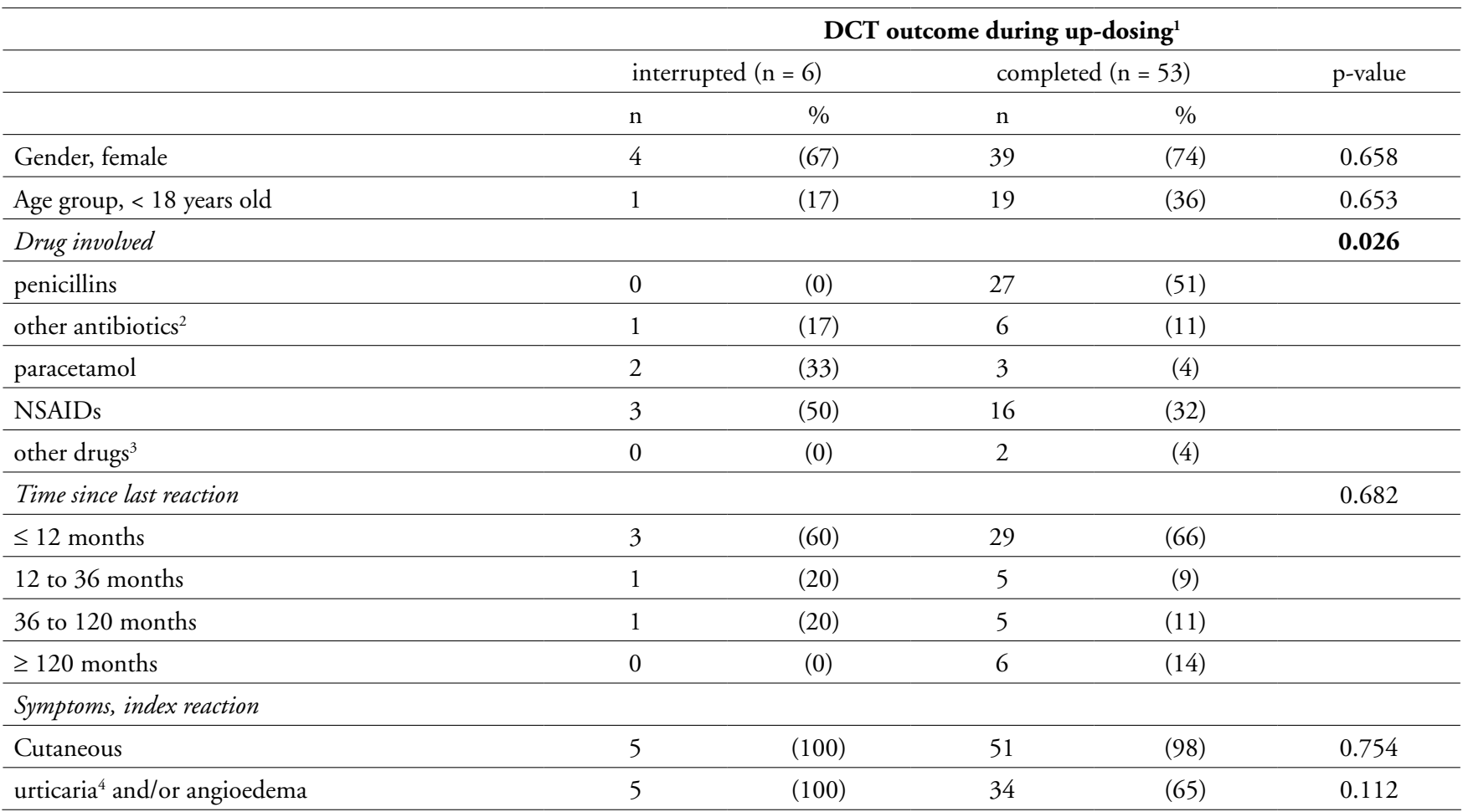


Table III - (continued)

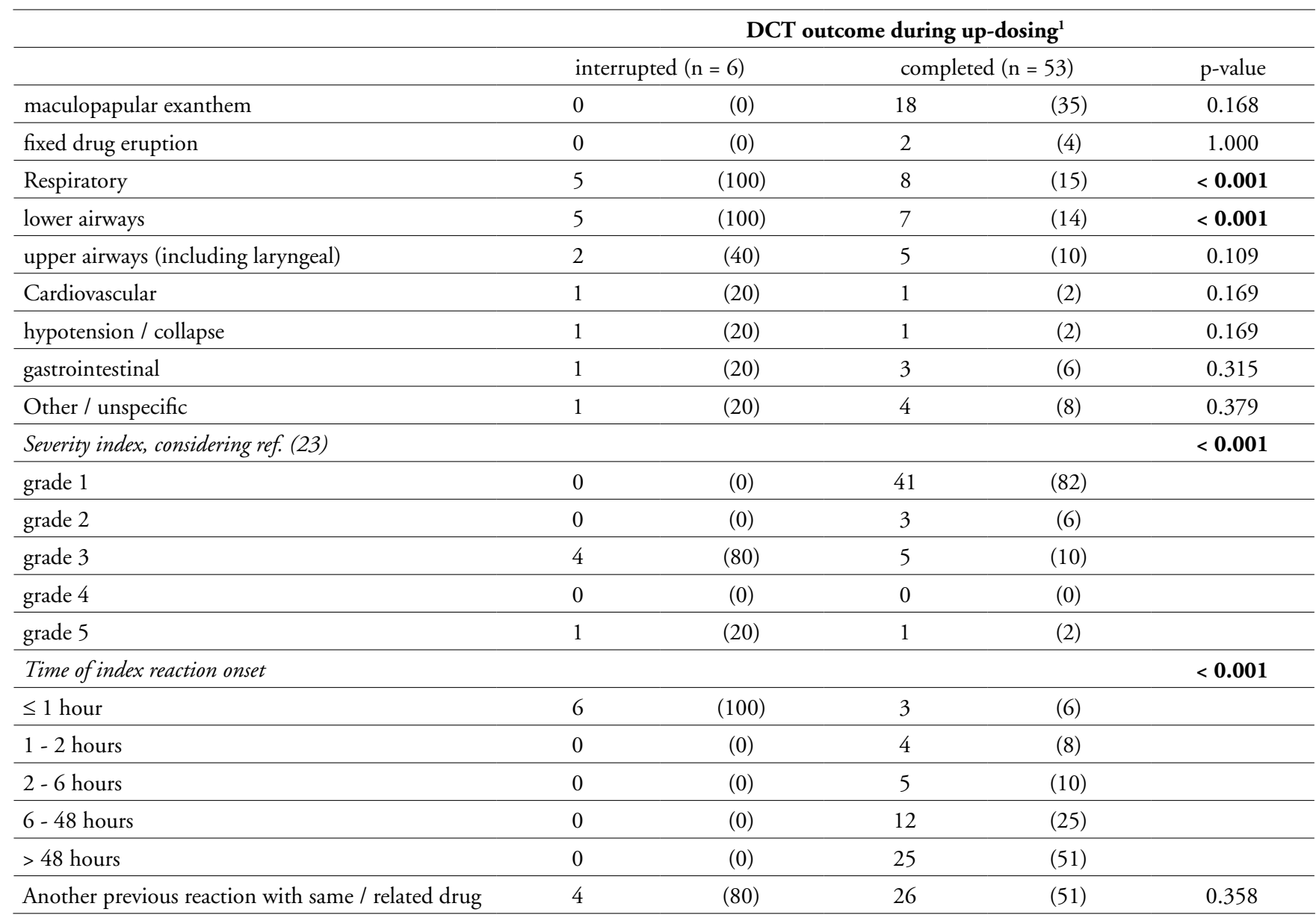

${ }^{1}$ The up-dosing phase of the DCT was considered completed if the target cumulative dose was reached; ${ }^{2}$ Including cephalosporins, ciprofloxacin, cotrimoxazole, levofloxacin, minocycline, nitrofurantoin, vancomycin; ${ }^{3}$ Including albendazole, atropine, betamethasone, cisatracurium, influenza vaccine, patent blue; ${ }^{4}$ Includes urticaria and/or erythema-warmth and/or pruritus, other than localized at the injection site (23). NSAID, nonsteroidal anti-inflammatory drug.

as possible predictors. CART identified the timing $(\leq 1$ hour vs. $>1$ hour) and severity (grade $\leq 2$ vs. grade $\geq 3$ ) of the index reaction as the most important predictors of DCT outcome during up-dosing (figure 1). The final decision tree presented a classification accuracy of $98.3 \%$, with only one patient misclassified; all patients that had the DCT interrupted during up-dosing were correctly identified.

\section{Discussion}

In this study, the prevalence of ST or DCT-based allergy diagnosis was $15 \%$. Most index reactions were mild, presenting only cutaneous symptoms and beginning more than one hour after drug intake. Seventy-eight percent of the diagnoses were based on DCT. All DCTs were performed using multistep protocols with at least three predicted steps; the number of DCT steps was associated with the drug tested but not with symptoms, severity or time of index reaction onset. Although most DCT reactions were mild, $11 \%$ were more severe than reported for the index reaction. Only $10 \%$ of the DCTs were considered positive and interrupted during up-dosing; in about $70 \%$ of the DCTs the reaction begun at least 5 hours after the last supervised drug intake. The timing ( $\leq 1$ hour vs. $>1$ hour) and severity (grade $\leq$ 2 vs. grade $\geq 3$ ) of the index reaction were the most important predictors of DCT outcome during up-dosing.

\section{Strengths and limitations}

To our knowledge, this is the first study specifically assessing predictors of DCT outcome during the up-dosing phase. Having a good prediction rule for the outcome of the supervised up-dosing phase of DCT can support the development of short- 
Figure 1 - Decision tree for DCT outcome during up-dosing (interrupted vs. completed).

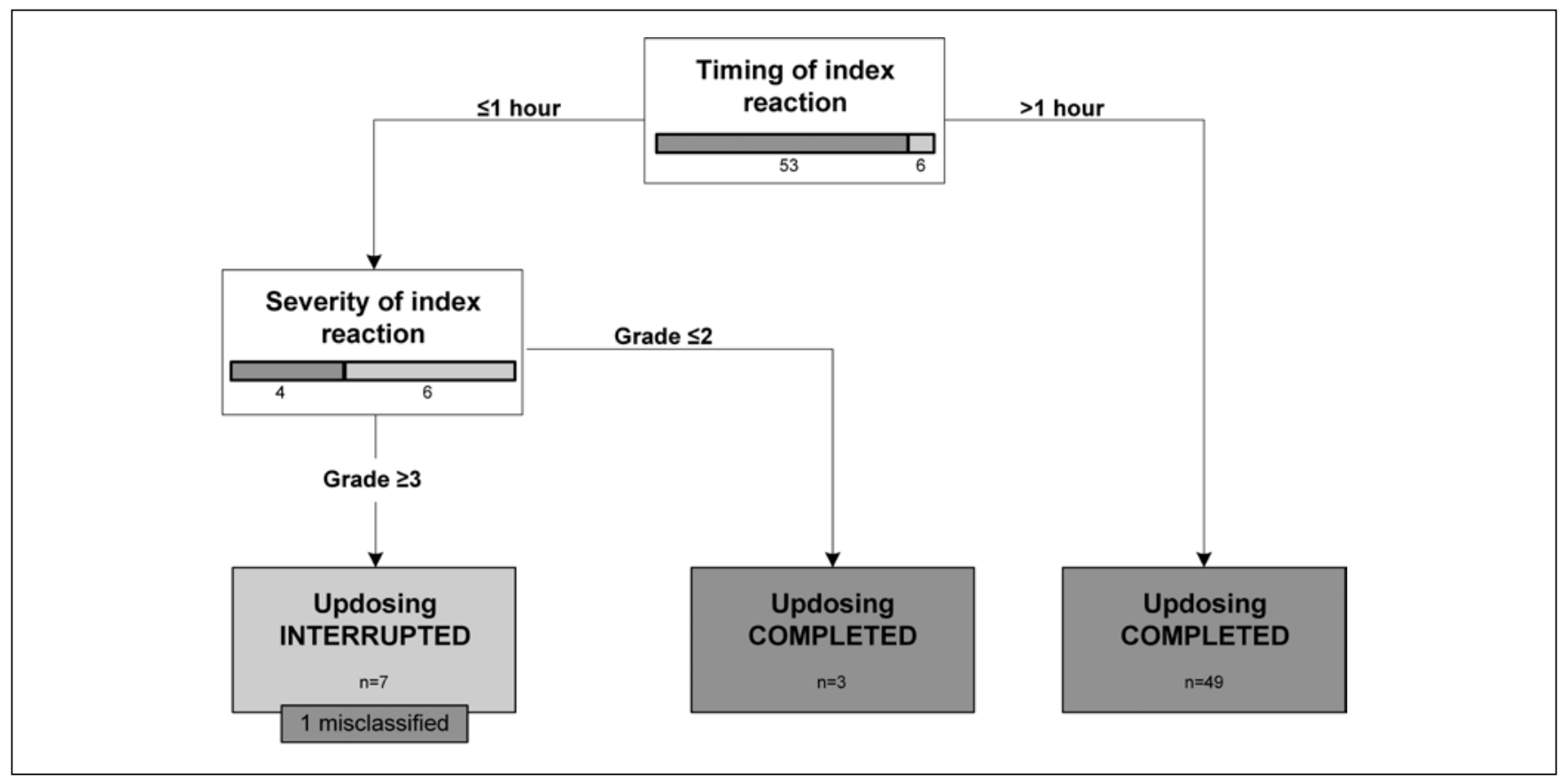

er and personalized DCT protocols in patients with low risk of reaction, decreasing the cost and time of these diagnostic procedures without increasing the risk of higher severity DCT reactions. However, this study is limited by the retrospective nature, with data collected from electronic and paper-based data recording systems. Moreover, the small sample size, especially in the analyses using comparisons between groups of DCT outcome and CART, makes these results only exploratory and in need of careful interpretation. The selection of patients with confirmed drug allergy, which would not be possible before diagnostic assessment and is inverted comparing to real-life, makes this study less directly applicable to clinical practice. However, we considered that it might be more effective to describe and evaluate predictors of DCT outcome in patients that were at "true" risk of interrupting DCT due to reaction. The inclusion of all patients, irrespective of the results of diagnostic assessments, although closer to the usual diagnostic reasoning, would increase the noise and decrease the focus in those patients. Nevertheless, before clinical use, these results need validation in a more comprehensive sample including patients with and without confirmed drug allergy.

The inclusion of patients presenting mostly mild reactions and predominantly with betalactams might also influence our results. Yet, these were the available patients in our centre and we believe they represent the usual clinical practice in most allergy units.
Interpretation of study findings and comparison with the literature

Previous studies, in various settings and populations, with diverse drugs and based on different diagnostic approaches, showed a prevalence of confirmed drug allergy in subjects with suspected drug reactions ranging from 3 to $27 \%$ (28-31). Our prevalence is higher than those reported in general settings (around 6\%) $(28,31)$, but is in line with a few studies held in specialized allergy units (30).

In our study, most patients presented the last reaction to the culprit drug within the previous 12 months. This puts most patients within the best time interval to perform the diagnostic assessment of suspected drug allergies. Indeed, a loss of sensitivity to drugs over time has been reported for IgE-mediated reactions (32), and after a time-interval of more than 6-12 months, some drug tests may already give negative results. Moreover, when the time interval between the reaction and the allergy assessment is longer, history is often less reliable and there is a lack of accurate information: the chronology is imprecise, the clinical manifestations are heterogeneous, making drug causality assessment more difficult to ascertain $(6,33)$. The short time interval between the index reaction and diagnostic assessment might have contributed to the low number of patients with missing data regarding the characteristics of the previous reactions. However, the agreement between the characteristics of the index and DCT reactions was only fair, especially regarding cardiovascular, gastrointestinal and 
specific cutaneous symptoms, suggesting that symptoms may be interpreted differently at different times/by different assessors or that DCT reaction might vary from the index reaction, even when it is recent; these findings are in line with another recent study (34). Nevertheless, most patients presented DCT reactions of similar severity as the index.

There are several difficulties regarding the choice of the best diagnostic approach to a specific patient, namely when to perform ST and which DCT protocol should be used. One of the controversies is related to the cut-off that should be used to differentiate immediate from nonimmediate reactions (2-5). In our study we chose to assess drug allergy diagnoses without a priori stratification by timing of the index reaction. This allowed us to explore the best cut-off to identify patients at higher risk of early DCT reaction ( $\leq 1$ hour vs. $>1$ hour), which, in our study, follows the classification proposed by Romano et al. (2).

In the past decade, several studies have evaluated prolonged DCTs to better detect nonimmediate DHRs with penicillin antibiotics (12-18). A recent study used a similar approach also in patients with non-severe immediate amoxicillin reactions beginning with an office-based supervised 3-steps DCT followed by a 4-day DCT; this study showed that this is a safe and effective way to rule out non-severe immediate and nonimmediate amoxicillin allergy, and ensures better compliance with future penicillin use (35). In that study, only $2.3 \%$ among the $130 \mathrm{pa}-$ tients who underwent a DCT presented a reaction on their initial visit (even if $20 \%$ had a suspected immediate reaction based on the clinical history) and all the remaining had to undergo a further ambulatory course of antibiotic continued at home (35). However, even in these studies, the diagnostic approach included a long, $\geq 3$ steps, office-based initial DCT. Only a few studies assessed the safety of shorter (1 or 2-step) DCT. Iammatteo M et al. (36) performed a retrospective study of 497 one or twostep test doses and compared the outcomes with those of multistep challenges. They included patients tested with several drug classes and found that one or two-step DCT were safe and that multistep challenges did not confer added safety. Mawhirt SL et al. (34) also reported that full dose challenges presented similar safety to multistep DCT in patients with immediate reactions to antibiotics. Our results, with most reactions occurring after the target dose was reached, irrespective of the tested drug, sup-

\section{References}

1. Johansson SG, Bieber T, Dahl R, Friedmann PS, Lanier BQ, Lockey RF, et al. Revised nomenclature for allergy for global use: Report of the Nomenclature Review Committee of the World Allergy Organization, October 2003. J Allergy Clin Immunol 2004; 113(5):832-836.

2. Romano A, Torres MJ, Castells M, Sanz ML, Blanca M. Diagnosis and management of drug hypersensitivity reactions. J Allergy Clin Immunol 2011; 127(Suppl3):S67-S73. port that these shorter DCT/test doses might be effective and safe way to diagnose drug allergy, at least in some patients.

In fact, DCT are long and expensive (9-11) diagnostic procedures that would benefit from a better patient stratification through the identification of predictors of early DCT reaction. We found timing and severity of index reactions as the best predictors of DCT outcome during up-dosing. Indeed, timing and severity of the index reaction are traditionally used to stratify diagnostic procedures to perform in patients with suspected drug allergy $(3,4,10)$. However, besides controversial classifications, there are no clearly defined cut-offs and practical decision rules to apply when selecting the diagnostic approach to follow in a specific patient. Our CART analysis, although exploratory, allowed the development of a decision tree that could be used to correctly identify patients that had the DCT interrupted. Our results suggest that, in most patients, DCT protocol could be more adapted to the patient and index reaction characteristics and less dependent on the drug. However, further studies comparing multistep with full dose or two-steps DCT protocols are warranted. The predictors of DCT outcome during up-dosing, found in our exploratory analysis, should be further tested in a different sample and validated into a prospective comparative study in real-life conditions.

\section{Conclusions}

Most drug allergy diagnoses were based on drug challenges, performed with multistep protocols dependent on the culprit drug. Only one tenth of the challenge reactions occurred during the up-dosing phase. The predictors of DCT interruption during up-dosing identified in the CART analysis can support the development of more personalized DCTs protocols but need further research before being applied into clinical practice.

\section{Acknowledgements}

We thank prof. João Fonseca for the inputs and critical review of the manuscript.

\section{Conflict of interests}

The authors declare that they have no conflict of interest.

3. Macy E, Romano A, Khan D. Practical Management of Antibiotic Hypersensitivity in 2017. J Allergy Clin Immunol Pract 2017; 5(3):577-586.

4. Demoly P, Adkinson NF, Brockow K, Castells M, Chiriac AM, Greenberger PA, et al. International Consensus on drug allergy. Allergy 2014; 69(4):420-437.

5. Levine BB. Immunologic Mechanisms of Penicillin Allergy. N Engl J Med 1966; 275(20):1115-1125. 
6. Dona I, Caubet JC, Brockow K, Doyle M, Moreno E, Terreehorst I, et al. An EAACI task force report: recognising the potential of the primary care physician in the diagnosis and management of drug hypersensitivity. Clin Transl Allergy 2018; 8:16.

7. Ryan D, van Weel C, Bousquet J, Toskala E, Ahlstedt S, Palkonen $S$, et al. Primary care: the cornerstone of diagnosis of allergic rhinitis. Allergy 2008; 63(8):981-989.

8. Aberer W, Bircher A, Romano A, Blanca M, Campi P, Fernandez J, et al. Drug provocation testing in the diagnosis of drug hypersensitivity reactions: general considerations. Allergy 2003; 58(9):854-863.

9. Blumenthal KG, Li Y, Banerji A, Yun BJ, Long AA, Walensky RP. The Cost of Penicillin Allergy Evaluation. J Allergy Clin Immunol Pract 2018; 6(3):1019-1027.e2.

10. Drug allergy: an updated practice parameter. Ann Allergy Asthma Immunol 2010; 105(4):259-273.

11. Iammatteo M, Alvarez Arango S, Ferastraoaru D, Akbar N, Lee AY, Cohen HW, et al. Safety and Outcomes of Oral Graded Challenges to Amoxicillin without Prior Skin Testing. J Allergy Clin Immunol Pract 2019;7(1):236-243.

12. Confino-Cohen R, Rosman Y, Meir-Shafrir K, Stauber T, Lachover-Roth I, Hershko A, et al. Oral Challenge without Skin Testing Safely Excludes Clinically Significant Delayed-Onset Penicillin Hypersensitivity. J Allergy Clin Immunol Pract 2017; 5(3):669-675.

13. Mendelson LM, Ressler C, Rosen JP, Selcow JE. Routine elective penicillin allergy skin testing in children and adolescents: study of sensitization. J Allergy Clin Immunol 1984; 73(1 Pt 1):76-81.

14. Hjortlund J, Mortz CG, Skov PS, Bindslev-Jensen C. Diagnosis of penicillin allergy revisited: the value of case history, skin testing, specific IgE and prolonged challenge. Allergy 2013; 68(8):1057-1064.

15. Hjortlund J, Mortz CG, Skov PS, Eller E, Poulsen JM, Borch JE, et al. One-week oral challenge with penicillin in diagnosis of penicillin allergy. Acta Derm Venereol 2012; 92(3):307-312.

16. Borch JE, Bindslev-Jensen C. Full-course drug challenge test in the diagnosis of delayed allergic reactions to penicillin. Int Arch Allergy Immunol 2011; 155(3):271-274.

17. Ponvert C, Perrin Y, Bados-Albiero A, Le Bourgeois M, Karila C, Delacourt $\mathrm{C}$, et al. Allergy to betalactam antibiotics in children: results of a 20-year study based on clinical history, skin and challenge tests. Pediatr Allergy Immunol 2011; 22(4):411-418.

18. Blanca-Lopez N, Zapatero L, Alonso E, Torres MJ, Fuentes V, Martinez-Molero MI, et al. Skin testing and drug provocation in the diagnosis of nonimmediate reactions to aminopenicillins in children. Allergy 2009; 64(2):229-233.

19. Demoly P, Kropf R, Bircher A, Pichler WJ. Drug hypersensitivity: questionnaire. EAACI interest group on drug hypersensitivity. Allergy 1999; 54(9):999-1003.

20. Brockow K, Romano A, Blanca M, Ring J, Pichler W, Demoly P. General considerations for skin test procedures in the diagnosis of drug hypersensitivity. Allergy 2002; 57(1):45-51.

21. Mori F, Cianferoni A, Barni S, Pucci N, Rossi ME, Novembre E. Amoxicillin allergy in children: five-day drug provocation test in the diagnosis of nonimmediate reactions. J Allergy Clin Immunol Pract 2015; 3(3):375-380.e1.

22. Brockow K, Ardern-Jones M, Mockenhaupt M, Aberer W, Barbaud A, Caubet JC, et al. EAACI position paper on how to classify cutaneous manifestations of drug hypersensitivity. Allergy 2018.

23. Cox LS, Sanchez-Borges M, Lockey RF. World Allergy Organization Systemic Allergic Reaction Grading System: Is a Modification Needed? J Allergy Clin Immunol Pract 2017; 5(1):58-62.e5.

24. Cox L, Larenas-Linnemann D, Lockey RF, Passalacqua G. Speaking the same language: The World Allergy Organization Subcutaneous Immunotherapy Systemic Reaction Grading System. J Allergy Clin Immunol 2010; 125(3):569-574, 74.e1-74.e7.

25. Bircher AJ, Scherer Hofmeier K. Drug hypersensitivity reactions: Inconsistency in the use of the classification of immediate and nonimmediate reactions. J Allergy Clin Immunol 2012; 129(1):263-264.

26. Altman DG. Practical Statistics for Medical Research. London: Chapman and Hall/CRC; 1991.

27. Marshall RJ. The use of classification and regression trees in clinical epidemiology. J Clin Epidemiol 2001; 54(6):603-609.

28. Erkocoglu M, Kaya A, Civelek E, Ozcan C, Cakir B, Akan A, et al. Prevalence of confirmed immediate type drug hypersensitivity reactions among school children. Pediatr Allergy Immunol 2013; 24(2):160-167.

29. Thong BYH, Tan T-C. Epidemiology and risk factors for drug allergy. Br J Clin Pharmacol 2011; 71(5):684-700.

30. Gamboa PM. The epidemiology of drug allergy-related consultations in Spanish Allergology services: Alergologica-2005. J Investig Allergol Clin Immunol 2009; 19 Suppl 2:45-50.

31. Rebelo Gomes E, Fonseca J, Araujo L, Demoly P. Drug allergy claims in children: from self-reporting to confirmed diagnosis. Clin Exp Allergy 2008; 38(1):191-198.

32. Blanca M, Torres MJ, Garcia JJ, Romano A, Mayorga C, de Ramon E, et al. Natural evolution of skin test sensitivity in patients allergic to beta-lactam antibiotics. J Allergy Clin Immunol 1999; 103(5 Pt 1):918-924.

33. Benahmed S, Picot MC, Dumas F, Demoly P. Accuracy of a pharmacovigilance algorithm in diagnosing drug hypersensitivity reactions. Arch Intern Med 2005; 165(13):1500-1505.

34. Mawhirt SL, Fonacier LS, Calixte R, Davis-Lorton M, Aquino MR. Skin testing and drug challenge outcomes in antibiotic-allergic patients with immediate-type hypersensitivity. Ann Allergy Asthma Immunol 2017; 118(1):73-79.

35. Labrosse R, Paradis L, Lacombe J, Samaan K, Graham F, Paradis J, et al. Efficacy and Safety of 5-Day Challenge for the Evaluation of Nonsevere Amoxicillin Allergy in Children. J Allergy Clin Immunol Pract 2018;6(5):1673-1680.

36. Iammatteo M, Blumenthal KG, Saff R, Long AA, Banerji A. Safety and Outcomes of Test Doses for the Evaluation of Adverse Drug Reactions: A 5-Year Retrospective Review. J Allergy Clin Immunol Pract 2014; 2(6):768-774. 